BMJ Open

Diabetes

Research

\& Care

\title{
Correlates of second-line type 2 diabetes medication selection in the USA
}

\author{
Ronald T Ackermann, ${ }^{1,2}$ Amisha Wallia, ${ }^{1,2}$ Matthew J O'Brien, ${ }^{1,2}$ Raymond Kang, ${ }^{1,2}$ \\ Andrew Cooper, ${ }^{1,2}$ Margaret R Moran, ${ }^{1,2}$ David T Liss ${ }^{1,2}$
}

\begin{abstract}
To cite: Ackermann RT, Wallia A, O'Brien MJ, et al. Correlates of second-line type 2 diabetes medication selection in the USA. BMJ Open Diab Res Care 2017;5:e000421. doi:10.1136/ bmjdrc-2017-000421
\end{abstract}

- Additional material is published online only. To view please visit the journal online (http://dx.doi.org/10.1136/ bmjdrc-2017-000421).

Received 21 March 2017 Revised 3 July 2017 Accepted 10 August 2017

\section{(a) CrossMark}

${ }^{1}$ Department of Medicine, Northwestern University Feinberg School of Medicine, Chicago, Illinois, USA ${ }^{2}$ Institute for Public Health and Medicine, Northwestern University Feinberg School of Medicine, Chicago, Illinois, USA

Correspondence to Dr Matthew J 0'Brien; matthew.obrien1@ northwestern.edu

\section{ABSTRACT}

Objective Past research provides insufficient evidence to inform second-line diabetes medication prescribing when metformin is no longer sufficient. We evaluated patient, prescriber, and health plan characteristics associated with selection of second-line diabetes medications in the USA.

Research design and methods We used a multiple case-comparison study design to identify characteristics associated with the probability of starting each of six second-line diabetes medication alternatives within 77744 adults enrolled in commercial or Medicare Advantage health plans from 2011 to 2015. National administrative data were provided by a large commercial health payer. Multinomial logistic regression models were used to identify characteristics independently associated with selecting each diabetes drug class.

Results From 2011 to 2015, sulfonylureas still represented $47 \%$ of all second-line drug starts, with proportionately higher use in patients $\geq 75$ years of age (63\% of drug starts). Basal insulin was more likely to be selected when a past A1c test result was $>10 \%(13.0 \%$ vs $4.5 \%$ for those with $A 1 c<8 \% ; p<0.001)$. Initiation of a glucagon-like peptide-1 receptor agonist was associated with being female $(10.1 \%$ vs $6.0 \%$ for male; $p<0.001)$ and having a diagnosis code for obesity $(10.8 \%$ vs $6.9 \%$ for no diagnosis; $p<0.001)$. For all drug classes, the recent prescribing behavior of the provider was a strong correlate of subsequent second-line drug selection.

Conclusions Sulfonylureas continue to represent almost half of second-line diabetes medication starts in the USA. This could reflect overuse for some groups such as older adults, for whom some alternatives may be safer, although more costly and potentially less effective. Future research should compare outcomes of medication choices and conditions under which particular classes are most effective.

\section{INTRODUCTION}

Over the past two decades, there has been a sharp increase in the number of therapeutic drug classes to treat type 2 diabetes (T2D). ${ }^{1}$ Although metformin has remained a cornerstone of initial T2D management, most patients eventually require the addition of a second or third agent to achieve goals for glucose control. ${ }^{12}$ Unfortunately, there is insufficient past research to guide the optimal choice of second-line medication when metformin is no longer sufficient. ${ }^{3}$

\section{Significance of this study}

What is already known about this subject?

- Past research provides insufficient evidence to inform second-line diabetes medication prescribing when metformin is no longer effective.

What are the new findings?

- This study found that sulfonylureas remain the most commonly prescribed second-line diabetes drug class, despite the emergence of newer classes that do not cause weight gain or hypoglycemia.

- This pattern may reflect overuse of sulfonylureas for certain patient groups such as older adults, for whom some alternatives may be safer, although more costly.

How might these results change the focus of research or clinical practice?

- In general, provider characteristics and recent prescribing patterns showed strong associations with diabetes drug class selection, suggesting a need for further research on whether and how provider behavior can be influenced to promote evidence-based medication prescribing.

Current guidelines allow for shared clinical decision-making based on goals for glucose and body weight outcomes, avoidance of possible side effects such as hypoglycemia, drug costs, and patient preference. ${ }^{2}$ Such guidance is likely to yield high variation in treatment selection, which may be driven more by drug cost or a physician's past experience, rather than evidence for superior effectiveness. Little is currently known about the key determinants of diabetes medication prescribing in real-world practice.

This study was designed to evaluate the characteristics of patients, providers, and health plans that are associated with selection of the most common six classes of second-line T2D medications. Understanding treatment patterns and correlates of T2D medication selection is important because it can help identify possible reasons for overuse or underuse of particular medications. Moreover, this study will inform future research 


\begin{tabular}{|c|c|c|c|c|c|c|}
\hline & DPP4 & GLP1 & INS/B & SGLT2 & SFU & TZD \\
\hline First on market & 2006 & 2005 & $\begin{array}{l}\text { NPH } \\
\text { (isophane } \\
\text { insulin)1982; } \\
\text { Lantus 2000 }\end{array}$ & March 2013 & 1950s & 1997 \\
\hline Route & Oral & Injection & Injection & Oral & Oral & Oral \\
\hline $\begin{array}{l}\text { Mean A1c- } \\
\text { lowering }^{*}\end{array}$ & $0.25 \%-1.0 \%$ & $0.8 \%-1.5 \%$ & No limit & $0.5 \%-1.0 \%$ & $1.0 \%-1.5 \%$ & $0.5 \%-1.5 \%$ \\
\hline Hypoglycemia & No & No & Yes & No & Yes & No \\
\hline Cost & $\$ 428-\$ 436$ & $\$ 527-\$ 831$ & $\$ 165-\$ 355$ & $\$ 470$ & $\$ 50-\$ 94$ & $\$ 349-\$ 355$ \\
\hline Body weight & Neutral/loss & Loss & Gain & Loss & Gain & Gain \\
\hline Other cautions & $\begin{array}{l}\text { Kidney disease; } \\
\text { ketoacidosis; } \\
\text { pancreatitis }\end{array}$ & $\begin{array}{l}\text { Pancreatitis; } \\
\text { thyroid cancer; } \\
\text { gastrointestinal } \\
\text { upset }\end{array}$ & & $\begin{array}{l}\text { Urine and vaginal infection; } \\
\text { kidney disease; fractures; } \\
\text { caution elderly ketoacidosis }\end{array}$ & $\begin{array}{l}\text { Caution } \\
\text { elderly }\end{array}$ & $\begin{array}{l}\text { congestive heart } \\
\text { failure; liver } \\
\text { disease; edema; } \\
\text { fractures }\end{array}$ \\
\hline
\end{tabular}

${ }^{*}$ Range of mean $\mathrm{A} 1 \mathrm{c}$ reductions from synthesis of multiple trials. ${ }^{9}$

†Range of median wholesale prices for monthly supply. ${ }^{10}$

DPP4, dipeptidyl peptidase-4 inhibitors; GLP1, glucagon-like peptide-1 receptor agonists; INS/B, long-acting or intermediate-acting insulin given as a basal (rather than mealtime) injection; SGLT2, sodium-glucose cotransporter 2 inhibitors; SFU, sulfonylurea or meglitinides; TZD, thiazolidinediones.

that evaluates the comparative effectiveness of different diabetes treatment options on glycemic control, diabetes complications, potential adverse treatment outcomes, and direct medical expenditures.

\section{RESEARCH DESIGN AND METHODS}

\section{Study design and exposures of interest}

Using administrative databases including more than 53 million health plan enrollees, we applied a multiple case-comparison study design to evaluate correlates of a first fill for one of six second-line T2D medication classes received by patients who previously were prescribed metformin alone. The six diabetes drug classes compared were dipeptidyl peptidase-4 (DPP4) inhibitors, glucagon-like peptide-1 (GLP1) receptor agonists, long-acting or intermediate-acting insulin given as a basal (rather than mealtime) injection (INS/B), sodium-glucose cotransporter 2 (SGLT2) inhibitors, sulfonylurea or meglitinides (SFU), and thiazolidinediones (TZD). An overview of these medications is displayed in table 1 . Because this research used coded, non-identifiable data, the Northwestern University Institutional Review Board judged that this work was not classifiable as human subjects research.

\section{Study population}

The study included health plan enrollees nationally who were $\geq 18$ years of age and had diabetes treated with metformin alone before recording a first fill for a second-line diabetes medication. Individuals who met the following three criteria were considered to have T2D: (1) $\geq 1$ pharmacy claim for one of the six diabetes medication classes of interest (we refer to the first dispensing date as the 'index date'); (2) $\geq 1$ inpatient or outpatient medical claim with a diabetes diagnosis code occurring on or before the index date; and (3) $\geq 1$ pharmacy claim for metformin in the 180 days before the index date. We excluded patients who did not also have evidence of metformin pharmacy claims in the 180 days after the index date. In addition, we excluded patients with a fill for any other T2D medication before the index date. Finally, we excluded patients with evidence of a pregnancy or a condition or treatment that might cause secondary diabetes, including hemochromatosis, acromegaly, cystic fibrosis, or more than 21 days of an oral corticosteroid medication within 180 days of the index date. Details regarding the definitions for these criteria are available in the online supplementary appendix.

\section{Measures and outcomes}

The primary study outcome was the probability of a first prescription fill for a drug in each of the six T2D medication classes. We explored associations with independent variables believed to be related to the likelihood of prescribing a particular drug class. Patient-level characteristics included gender, age, race/ethnicity, Charlson Comorbidity Score, ${ }^{4}$ and presence/absence of a diagnosis code for obesity; most recent hemoglobin Alc test result (categorized as $<8 \%, 8 \%-9.9 \%, \geq 10 \%$, or 'no value available'); census region of the individual's home zip code; and indicators of utilization within 90 days of the index date that might influence medication choice (eg, presence/absence of a diagnosis of hypoglycemia; presence/absence of a hospitalization; presence/ absence of a diagnosis of poorly controlled diabetes; total patient out-of-pocket expenditure). Provider-level or 'prescriber'-level variables included specialty type (family physician, general internist, endocrinologist, 
nurse practitioner, or 'other'); a prescribing index reflecting the percentage of a provider's patients who have diabetes; and indicators for high versus low probability of recent prescribing of each of the six drug classes (based on the percent of a provider's total T2D medication prescriptions that were for DPP4s, GLP1s, INS/ Bs, SFUs, SGLT2s, or TZDs). Health plan-level variables included an index of the 'richness' of health coverage (ie, the median value of the percentage of total healthcare costs paid by the patient, for all persons in the same health plan); an indicator variable for enrollment in a Medicare versus commercial health plan; and the type of plan design (health maintenance organization (HMO); preferred provider organization (PPO); point of service (POS); indemnity (ie, traditional fee for service); exclusive provider organization; or other plan type). Please see the online supplementary appendix for additional details.

\section{Data sources}

Data sources included health plan enrollment files, medical inpatient and ambulatory claims, and pharmacy claims. Although most patients had recent laboratory claims for A1c tests, test results are typically not available from administrative data. However, the laboratory claims file did include results for $36 \%$ of A1c claims. The availability of these results was comparable across the six T2D medication classes. Information about race and ethnicity also was not consistently available in these administrative data but was imputed by the data vendor using a mix of individual-level and neighborhood-level characteristics.

\section{Statistical analysis}

Descriptive statistics for baseline patient, provider, and plan characteristics were summarized across each of the six drug classes. Student's t-tests were used to compare continuous variables; $\mathrm{X}^{2}$ tests were used to compare categorical variables. Multinomial logistic regression models were used to identify variables that were independently associated with the odds of selecting a medication from each diabetes drug class. All patient-level, prescriber-level, and health plan-level variables were included as covariates in each model. We present the predicted probabilities produced by marginal standardization, where the probabilities are proportionally adjusted according to the weight for each level of the confounding factors. ${ }^{5}$

\section{RESULTS}

\section{Prescribing volume and patterns of select diabetes}

\section{medications}

Between January 2011 and June 2015, 77744 patients had evidence of metformin monotherapy followed by a first fill for one of the six second-line T2D drug classes. SFUs were prescribed most frequently, accounting for almost half $(47.4 \%)$ of all prescriptions $(98.9 \%$ of which were sulfonylureas; $1.1 \%$ were meglitinides); TZDs were prescribed least, accounting for $<5 \%$ of all prescriptions. However, the relative contribution of each drug class to total prescribing changed considerably over the 5-year period (figure 1). Between 2011 and 2012, DPP4 prescribing increased from $19 \%$ to $32 \%$ of overall secondline drug starts, with concomitant reductions in both SFUs and TZDs. Since entering the market in 2013, the

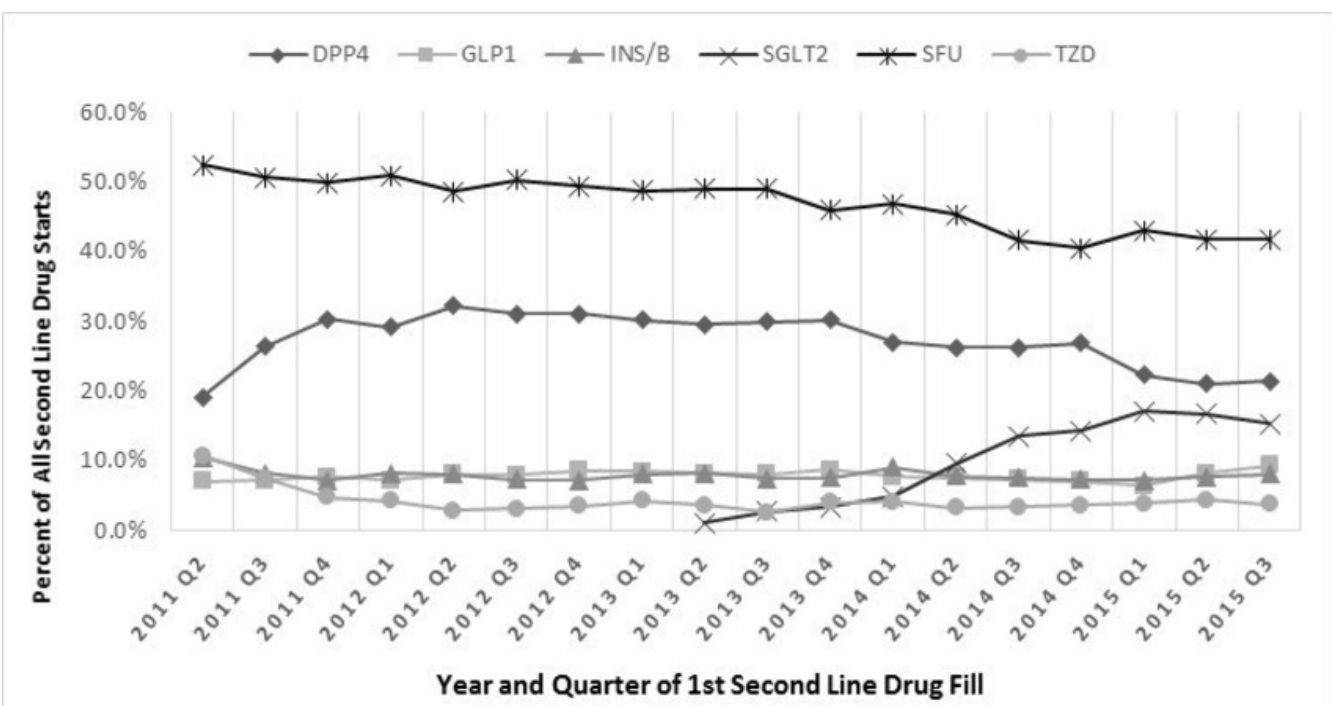

Figure 1 Trends in the relative contribution of each second-line diabetes medication class, by quarter, as a percent of all second-line prescribing, 2011-2015. Each line represents a quarterly time trend for the percent of all second-line drug starts (ie, all six categories combined) that were contributed by each class; values for all six classes in each quarter total to $100 \%$. DPP4, dipeptidyl peptidase-4 inhibitors; GLP1, glucagon-like peptide-1 receptor agonists; INS/B, long-acting or intermediateacting insulin given as a basal (rather than mealtime) injection; SGLT2, sodium-glucose cotransporter 2 inhibitors; SFU, sulfonylurea or meglitinides; TZD, thiazolidinediones. 
percentage of overall prescribing attributable to SGLT2s increased from $0 \%$ to about $15 \%-17 \%$, with concomitant decreases in every other drug class except TZDs.

Unadjusted percentages of drug prescribing across select patient, provider, and health plan characteristics are displayed in table 2. The online supplementary appendix also includes unadjusted values for all other covariates examined. Slightly more than $43 \%$ of the study population were women, about $60 \%$ were between ages 45 and 64 , and about $27 \%$ were believed to be black or Hispanic/Latino. About $85 \%$ of the population were in commercial health plans; $15 \%$ were in Medicare Advantage plans. Just more than half of plans had a POS structure, and 28\% were HMOs. A majority of prescriptions were written by either a family medicine $(44 \%)$ or general internal medicine $(32 \%)$ provider; only $7 \%$ of prescriptions were from an endocrinologist. There were statistically significant differences across the six drug classes in every patient, prescriber, and health plan characteristic examined.

\section{Adjusted probabilities of second-line medication prescribing for T2D}

Table 3 shows the fully adjusted prescribing probabilities for each of the six drug classes, both overall, and within strata of patient, prescriber, and health plan characteristics. In adjusted models, the rank ordering of T2D drug class prescribing, from highest to lowest probability, was similar to unadjusted findings: SFUs were the most common, followed by DPP4s, GLP1s, INS/Bs, SGLT2s, and TZDs.

\section{Adjusted associations of T2D medication prescribing with patient characteristics}

SFUs remained the most common drug class across all patient-level characteristics. However, several characteristics were associated with significant differences in the selection of each class. For example, men received a GLP1 less often than women (5.9\% vs $10.1 \%$, difference $-4.1 \%, 95 \%$ CI $(-4.7 \%$ to $-3.6 \%))$ but were more likely than women to receive SFUs or TZDs. With advancing age, there was an increase in DPP4 and SFU prescribing, with a decrease in GLP1 and INS/B prescribing; 11.8\% of persons $<35$ years of age received a GLP1, compared with only $5.6 \%$ of individuals $64-75$ years of age (difference $-6.2 \%, 95 \%$ CI $(-8.2 \%$ to $-4.2 \%))$ and only $3.4 \%$ of individuals $\geq 75$ years (difference $-8.4 \%, 95 \%$ CI $(-10.5 \%$ to $-6.3 \%)$ ). Recent hospitalization was associated with a higher probability of receiving INS/B (difference $5.3 \%$, $95 \%$ CI $(4.4 \%$ to $6.2 \%)$ ) but relatively lower probabilities of DPP4s, GLP1s, or SGLT2s. When compared with patients with a prior A1c $<8 \%$, patients with A1c values $\geq 10 \%$ had a higher probability of receiving INS/B (difference $8.5 \%, 95 \%$ CI $(6.4 \%$ to $10.7 \%)$ ) and SFUs (difference $7.0 \%, 95 \% \mathrm{CI}(3.7 \%$ to $10.3 \%)$, and had lower probabilities of receiving DPP4s, GLP1s, SGLT2s, or TZDs. Finally, having a prior obesity diagnosis code was associated with higher probabilities of receiving
GLP1s (difference $3.9 \%$, 95\% CI $(3.2 \%$ to $4.6 \%)$ ) and SGLT2s (difference $1.0 \%, 95 \%$ CI $(0.6 \%$ to $1.5 \%)$ ), with lower probabilities of INS/B, SFUs, or TZDs.

\section{Adjusted associations of T2D medication prescribing with provider characteristics}

When compared with patients who were prescribed their T2D medication by a family physician, those with an endocrinologist prescriber were more likely to receive INS/B $(10.1 \%$ vs $7.8 \%$, difference $2.3 \%, 95 \%$ CI $(0.7 \%$ to $3.8 \%)$ ) or GLP1s (10.6\% vs $7.2 \%$, difference $3.3 \%$, $95 \%$ CI $(2.0 \%$ to $4.6 \%))$ but were less likely to receive SGLT2s, SFUs, or TZDs. Similar differences in prescribing patterns were observed comparing general internists and endocrinologists. Even after adjusting for the prescriber's specialty type, providers who wrote T2D prescriptions for a higher percentage of their patients were more likely to prescribe GLP1s than were providers with proportionately lower rates of diabetes drug prescribing (difference between highest and lowest quartile, $4.2 \%, 95 \%$ CI (3.4\% to $5.0 \%$ ). Providers with a relatively high level of recent prescribing for a particular drug class had a higher probability of selecting a T2D medication within that same class. For example, patients who received their medication from a provider who was above the 75 th percentile of recent prescribing for DPP 4 s had a $41 \%$ probability of receiving a DPP4, compared with only $19 \%$ of patients seeing a provider with no recent DPP4 prescribing. Similar patterns were observed for all six classes. Finally, geographical location was associated with choice of drug class. For example, compared with other regions, patients seeing prescribers in the Northeast were more likely to receive a DPP4 and less likely to receive an SFU than were patients in all other regions.

\section{Adjusted associations of T2D medication prescribing with health plan characteristics}

After adjustment for other patient, prescriber, and health plan differences, individuals in a Medicare Advantage plan were more likely than those in a commercial health plan to receive INS/B (difference 3.1\%, 95\% CI $(1.5 \%$ to $4.6 \%)$ ) and SFUs (difference $5.1 \%, 95 \%$ CI (2.8\% to $7.4 \%)$ ), and were less likely to receive DPP4s, GLP1s, or SGLT2s. The 'richness' of health coverage (ie, percentage of total healthcare costs that were paid by the patient) was also associated with T2D drug selection; compared with patients in health plans in the lowest quartile of out-of-pocket costs, patients in plans in the highest quartile were more likely to receive DPP4s (difference $2.6 \%$, $95 \%$ CI $(1.1 \%$ to $4.1 \%))$ and were less likely to receive INS/B (difference $-1.1 \%, 95 \%$ CI $(-2.1 \%$ to $-0.3 \%)$ ) or SFUs (difference $-2.4 \%, 95 \%$ CI $(-4.0 \%$ to $-0.7 \%)$ ). No clear associations were observed with the health plan structure (eg, HMO vs PPO plans).

\section{CONCLUSIONS}

Despite the emergence of newer classes of T2D medications that do not cause weight gain or hypoglycemia, 


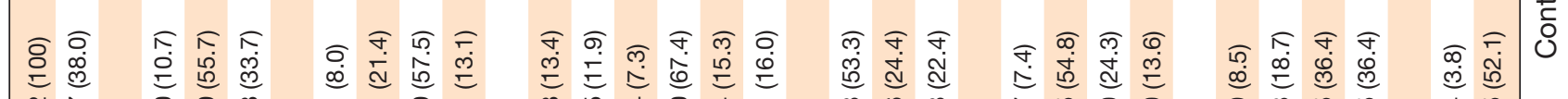

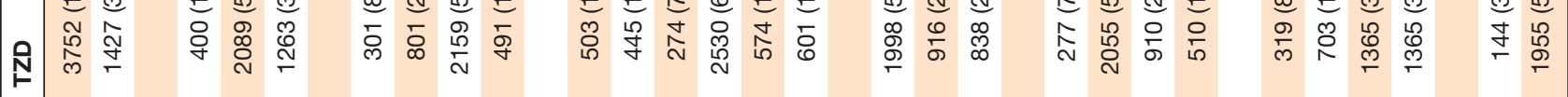

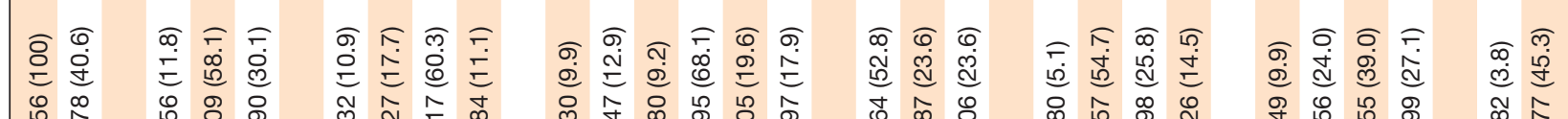

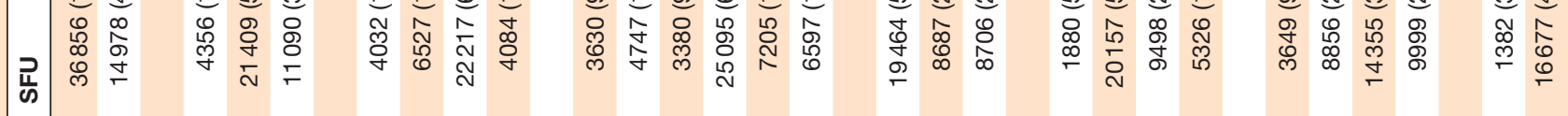

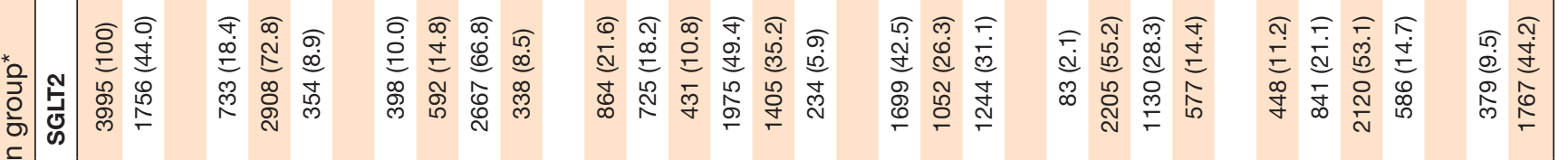
들

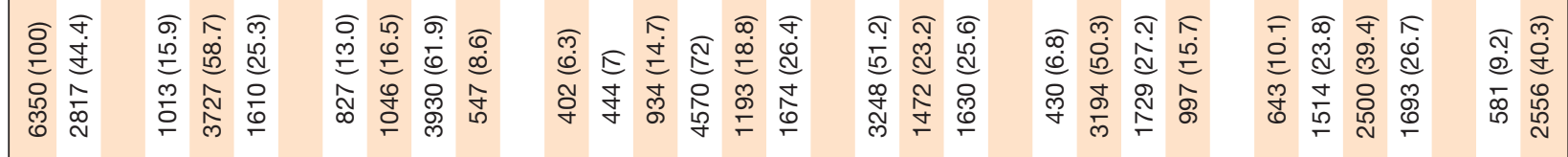

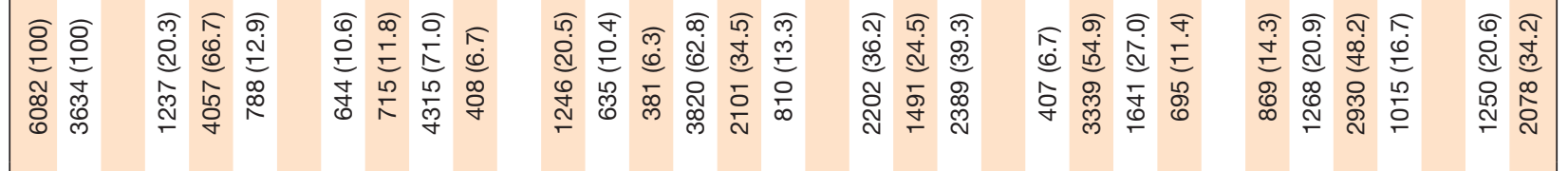

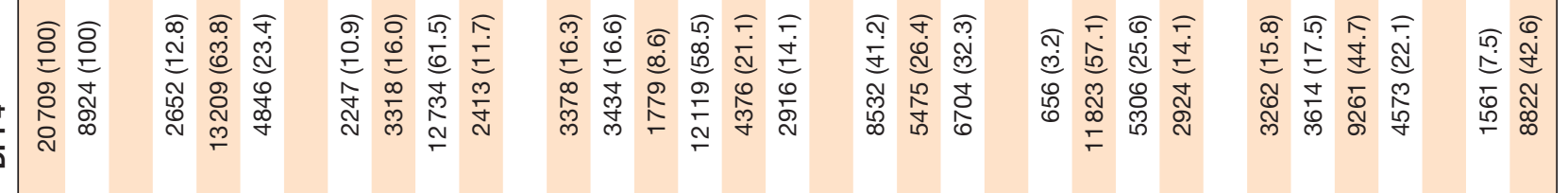

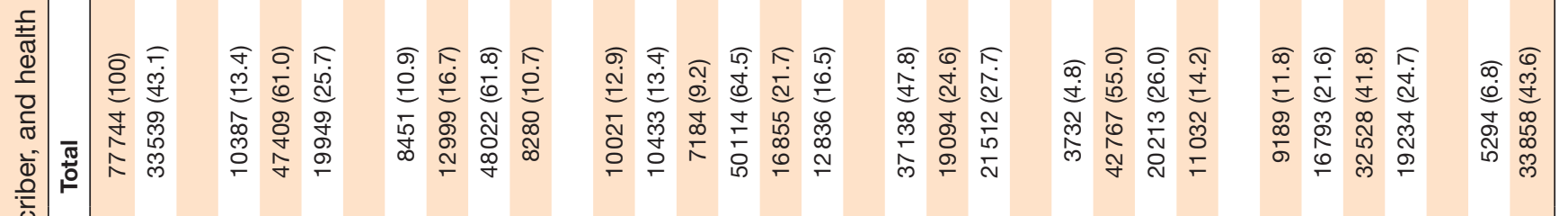

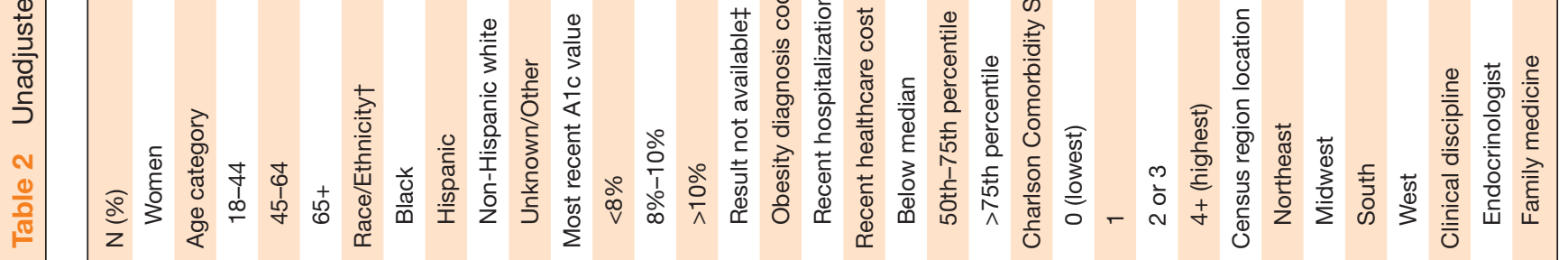




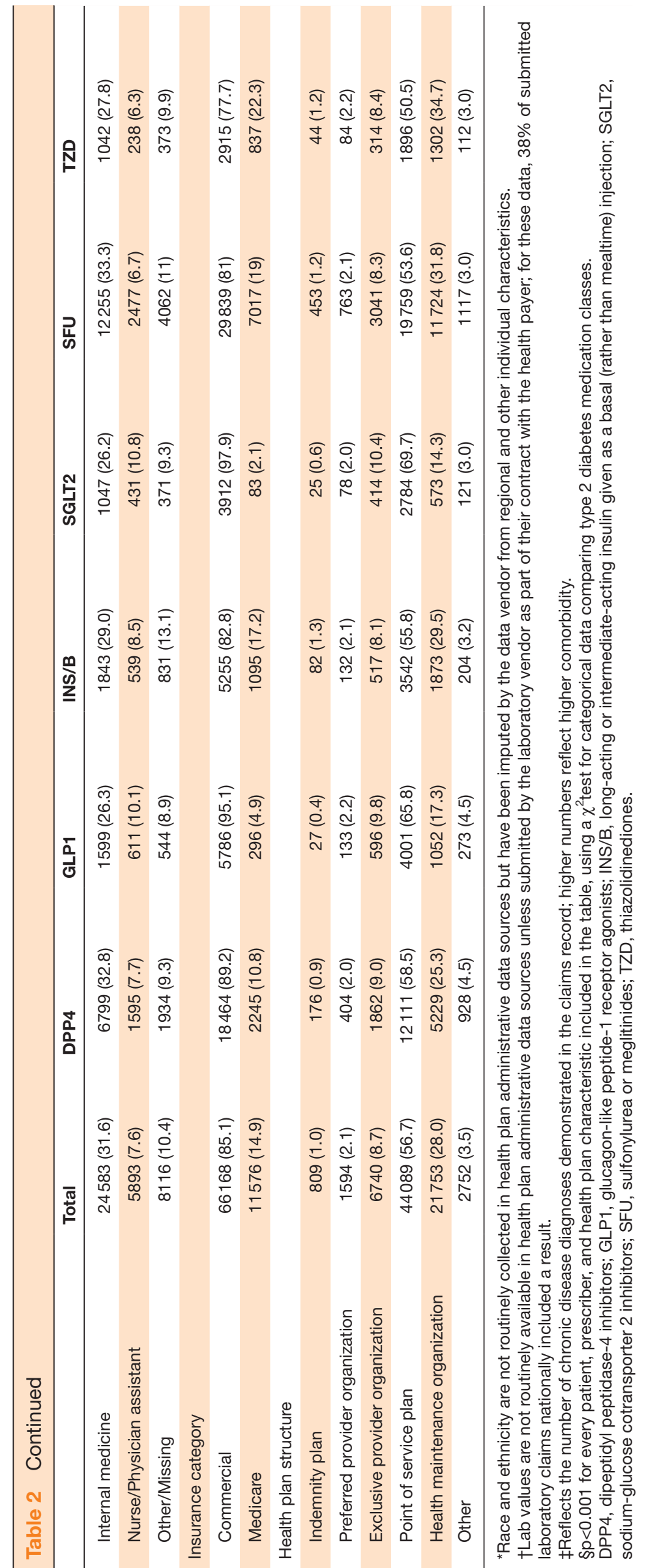




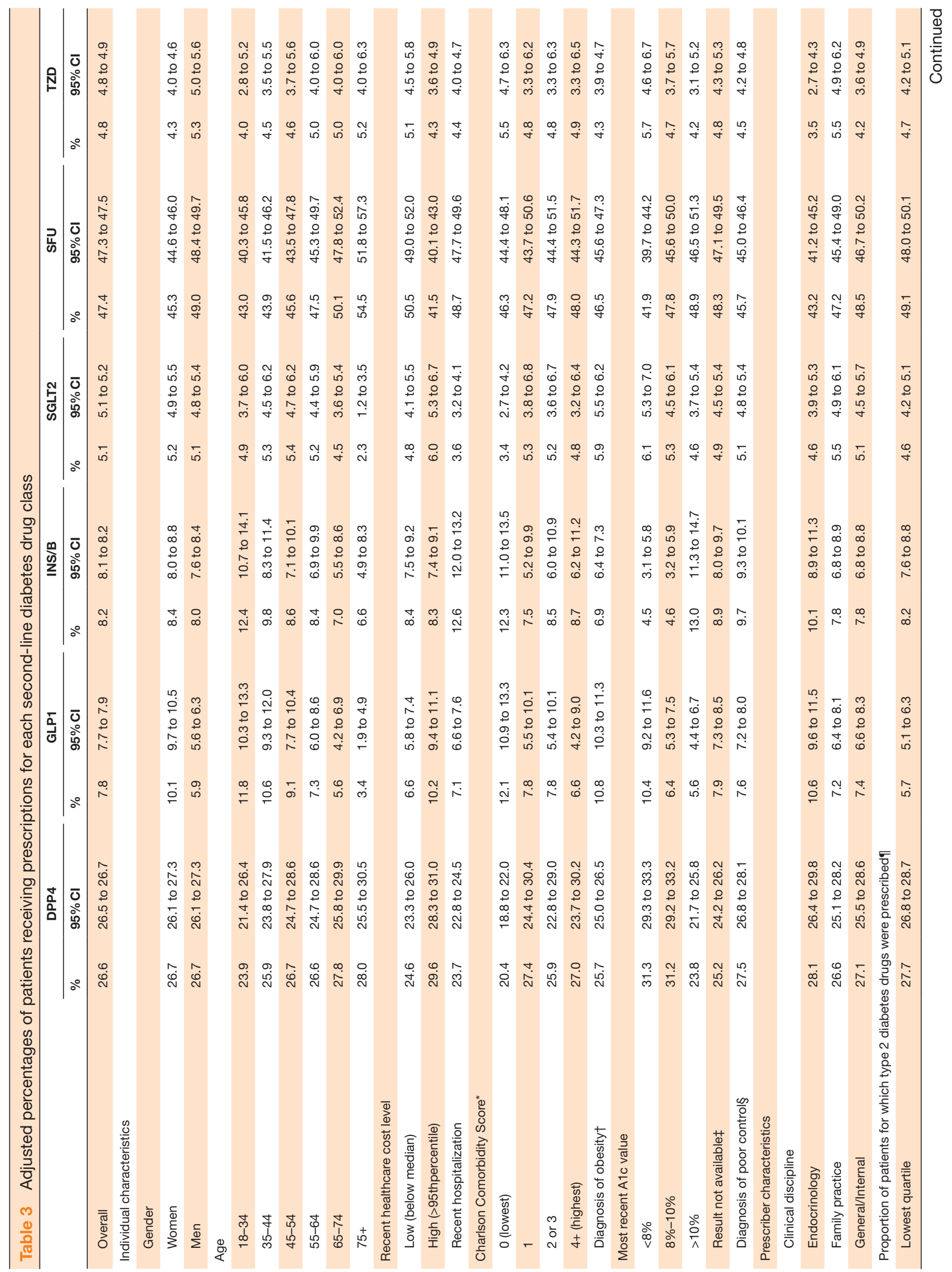




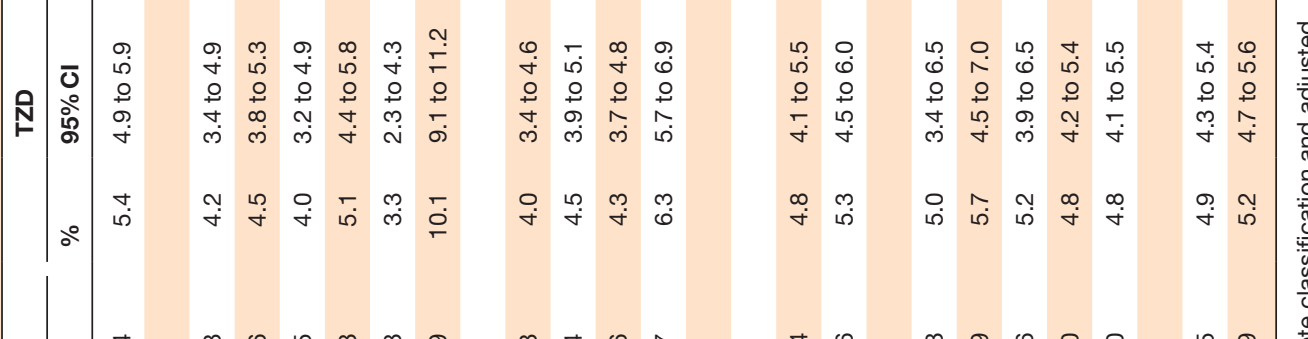

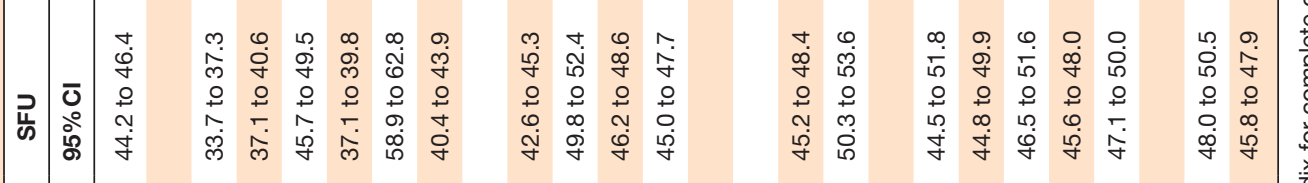

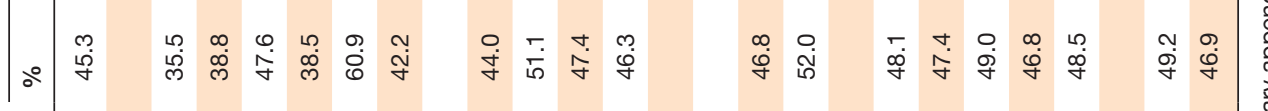

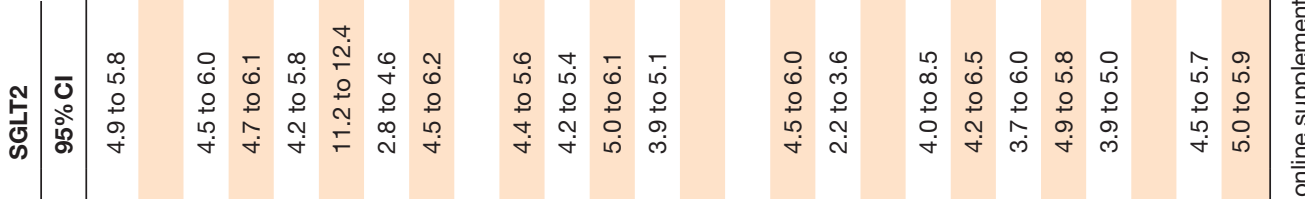

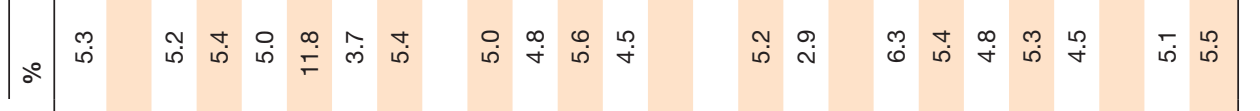

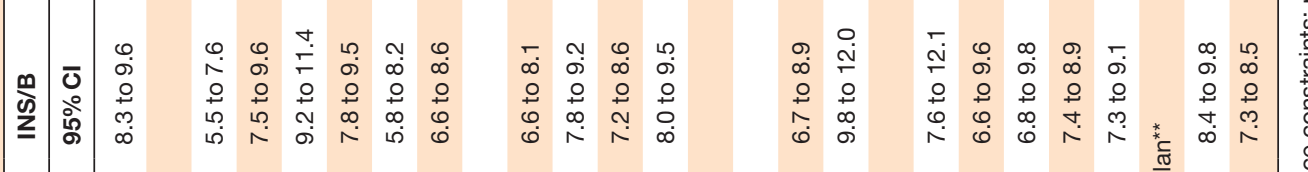

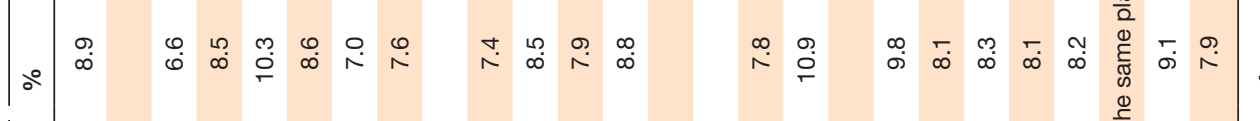

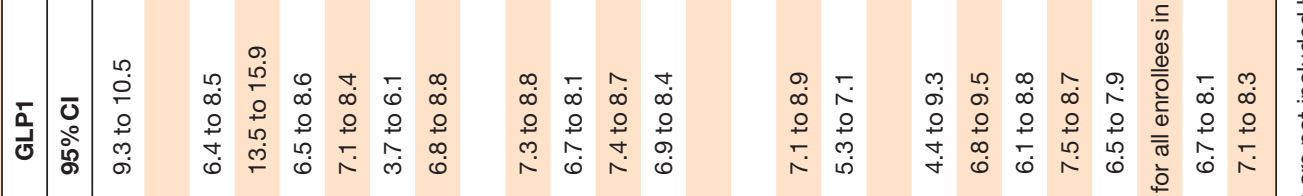

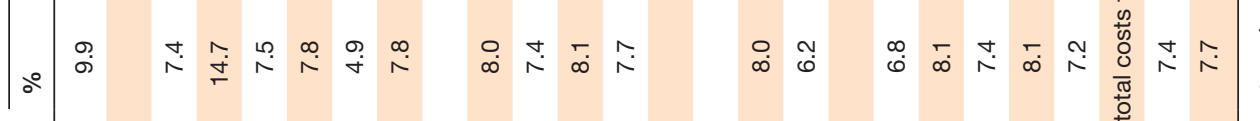

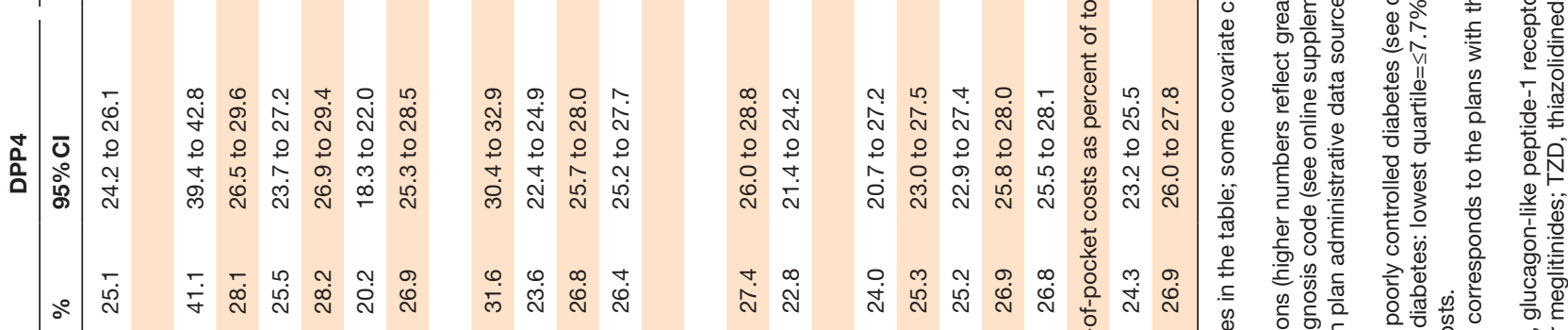

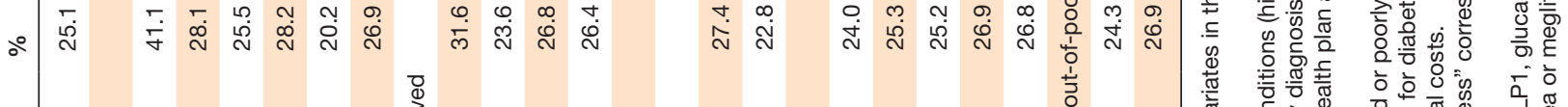

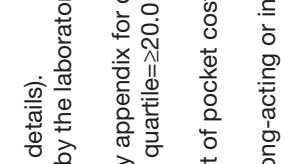

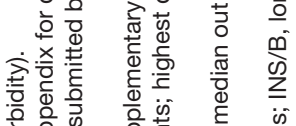

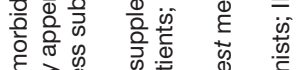

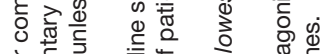

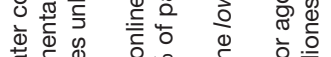

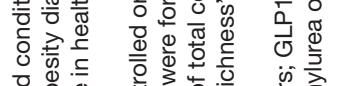

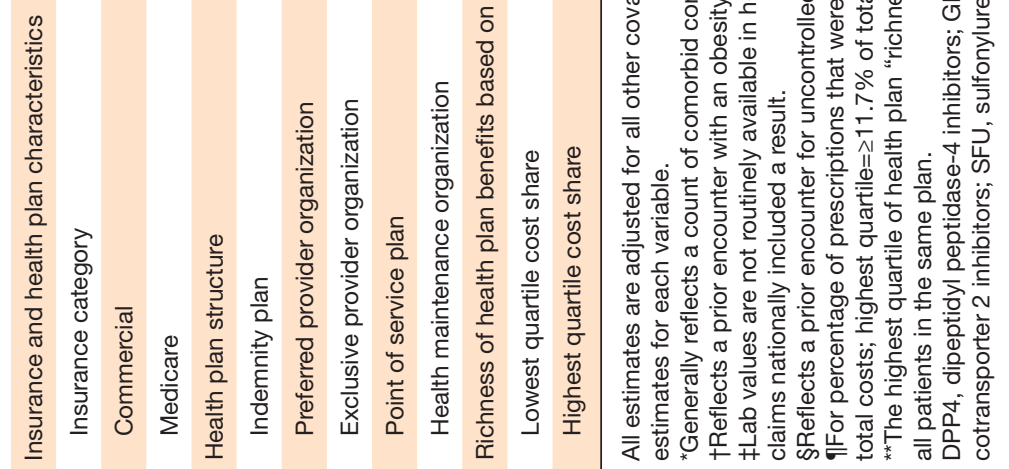

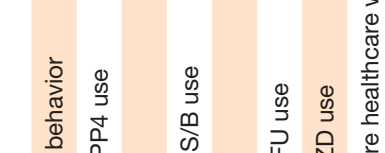

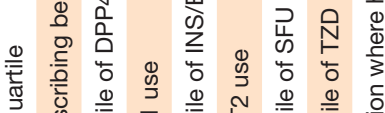

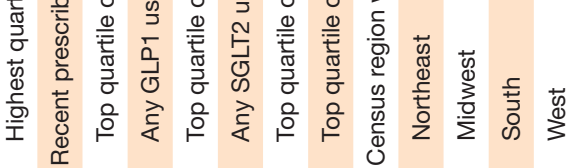


SFUs remain the most commonly prescribed second-line diabetes drug class. Since 2013, prescribing has increased most for SGLT2s, with corresponding decreases in every other drug class except TZDs. Although this demonstrates changes in T2D medication prescribing, SFUs were still selected for about $42 \%$ of patients in 2015. Because SFUs have been on the market since the 1950s, prescribers may have more familiarity with how to initiate these medications and more confidence that they are aware of potential side effects. SFUs also cost less and have greater glucose-lowering effects than do many newer medication alternatives, possibly also contributing to their sustained use.

Our analysis uncovered several associations among patient-level, prescriber-level, and health plan-level characteristics with the selection of second-line T2D medications. For example, SFU selection increased for patients who were over age 65 and those with higher past A1c results. Initiation of basal insulin increased for patients who were younger, non-obese, and who had a recent hospitalization or prior high Alc result. Initiation of a GLP1 was higher for patients who were younger, female, obese, or who had lower past A1c test results. Selection of DPP4s increased for patients $\geq 45$ years of age, as well as for those with higher comorbidity and lower past A1c results. SGLT2 selection increased for patients who were $<75$ years of age and who had lower past A1c results.

Many of these patterns seem appropriate and may reflect some amount of patient-centered prescribing. ${ }^{6}$ For example, SFUs and basal insulin have greater glucose-lowering efficacy but also have concerns for weight gain and hypoglycemia, which may diminish prescribing among patients who are obese or who have lower A1c results. By contrast, DPP4, GLP1, and SGLT2 agents are often viewed to have lower glucose-lowering efficacy, minimal threat of hypoglycemia, and do not promote weight gain, making them more favorable for patients who have lower A1c tests or are more concerned about weight gain. One unanticipated finding was that adults $>65$ years and those on Medicare plans were most likely to receive SFUs. Two of the drugs in this class (glyburide and chlorpropamide) are on the American Geriatric Society's Beers' list of medications to avoid because of safety concerns related to unpredictable and severe hypoglycemia in older adults. ${ }^{78}$ About $70 \%$ of new SFU drug starts among adults $>65$ years were for a long-acting SFUs, and $21 \%$ included one of the two drugs on the Beers' list; only $1.4 \%$ were for meglitinides, which generally have lower risk for hypoglycemia. This finding represents an opportunity for further research as well as for quality improvement initiatives to ensure safe prescribing of antiglycemic medications in older adults.

Our analysis found that a provider's recent prescribing of a particular drug class had a strong relationship with subsequent drug selection, suggesting that repetition and familiarity initiating a particular drug may be important determinants of drug selection. This may not be surprising, but it underscores the risk of industry activities to influence prescriber behavior in ways that may not be evidence-based, as well as the importance of quality assurance efforts that insure all prescribers have a balanced approach to prescribing. Another interesting pattern was the relationship between having proportionately higher levels of diabetes prescribing (or being an endocrinologist) and the greater use of GLP1s. Although one might speculate that providers with more patients with diabetes are simply more likely to prescribe newer medications, a similar pattern was not observed for SGLT2 inhibitors. One explanation for greater prescribing of injectable medications might also be that higher volume providers have more capacity to provide patients with additional education when starting these medications, as well as familiarity with how to prescribe injection supplies.

Patients in health plans with lower out-of-pocket costs were generally less likely to receive newer or costlier second-line medications, such as DPP4s, GLP1s, and SGLT2s, and were more likely to receive drugs that are generally cheaper or have been on the market longer, such as SFUs and basal insulin. It is possible that health plans with lower cost sharing are more likely to impose formulary restrictions or tiered medication copays that offer SFUs at a lower cost to patients, thereby driving selection of those medications. However, the lack of a clear relationship between T2D drug selection and health plan structure (eg, HMO vs PPO) may suggest that other forms of drug and utilization management strategies used by plans may yield inconsistent effects on prescribing. This deserves further research.

\section{Limitations}

Our study has notable limitations. First, the reliance on administrative data could lead to misclassification of the timing of second-line treatment. For example, some manufacturers offer coupons to promote the use of newer medications, which may make it difficult to determine the timing of a drug start based on the first submitted pharmacy claim. This also could result in underestimation of the prevalence of some newer medications. Another limitation is that we did not have information about provider characteristics, such as age or date of last board certification, which may relate to their selection of medications. Finally, the observational study design limits our ability to know if associations are causal; additional longitudinal research is needed to determine if more frequent use of certain medications has any impact, good or bad, on important health or economic outcomes.

Our study provides important information about the resilience of sulfonylureas and recent emergence of SGLT2 inhibitors as second-line agents for the management of T2D when metformin is no longer sufficient. In general, select health plan, prescriber, and patient characteristics were associated with the probability that a particular second-line medication was selected. This information should cause health plan administrators to 
think carefully about all potential effects of policies that restrict formularies or shift medication costs to patients. These findings also will be valuable for the design of future research that compares potential benefits and harms of these medications on important health and economic outcomes.

Acknowledgements The authors wish to acknowledge the UnitedHealth Group Enterprise Research and Development for ensuring access to the data.

Contributors RTA contributed to the study design, interpreted analytic results and drafted the manuscript; AC and RK contributed to the study design, managed and cleaned raw data received from the sponsor, conducted the analyses, interpreted the findings, and edited/revised the manuscript; AW, MJO, DTL and MRM contributed to the study design, interpreted the results and edited/revised the manuscript.

Funding This work was supported by United Healthcare Services.

Competing interests AW discloses that she has received research grant support from Eli Lilly and has done trial adjudication for Lexicon Therapeutics, both of which are unrelated to this work.

Patient consent The Northwestern University Institutional Review Board determined that this work was not classifiable as human subjects research. Patient consent was not required.

Provenance and peer review Not commissioned; externally peer reviewed.

Open Access This is an Open Access article distributed in accordance with the Creative Commons Attribution Non Commercial (CC BY-NC 4.0) license, which permits others to distribute, remix, adapt, build upon this work non-commercially, and license their derivative works on different terms, provided the original work is properly cited and the use is non-commercial. See: http://creativecommons.org/ licenses/by-nc/4.0/ (c) Article author(s) (or their employer(s) unless otherwise stated in the text of the article) 2017. All rights reserved. No commercial use is permitted unless otherwise expressly granted.

\section{REFERENCES}

1. American Diabetes Association. Standards of medical care in diabetes--2014. Diabetes Care 2014;37(Suppl 1):S14-S80.

2. Garber AJ, Abrahamson MJ, Barzilay JI, et al. Consensus statement by the American Association of Clinical Endocrinologists and American College of Endocrinology on the comprehensive type 2 diabetes management algorithm--2016 executive summary. Endocr Pract 2016;22:84-113.

3. Nathan DM, Buse JB, Kahn SE, et al. Rationale and design of the glycemia reduction approaches in diabetes: a comparative effectiveness study (GRADE). Diabetes Care 2013;36:2254-61.

4. Deyo RA, Cherkin DC, Ciol MA. Adapting a clinical comorbidity index for use with ICD-9-CM administrative databases. J Clin Epidemiol 1992;45:613-9.

5. Muller CJ, MacLehose RF. Estimating predicted probabilities from logistic regression: different methods correspond to different target populations. Int J Epidemiol 2014:43:962-70.

6. Reusch JE, Manson JE. Management of Type 2 Diabetes in 2017: Getting to Goal. JAMA 2017;317:1015-6.

7. Bodmer M, Meier C, Krähenbühl S, et al. Metformin, sulfonylureas, or other antidiabetes drugs and the risk of lactic acidosis or hypoglycemia: a nested case-control analysis. Diabetes Care 2008;31:2086-91.

8. Hamnvik OP, McMahon GT. Balancing risk and benefit with oral hypoglycemic drugs. Mt Sinai J Med 2009;76:234-43.

9. Sherifali D, Nerenberg K, Pullenayegum E, et al. The effect of oral antidiabetic agents on A1C levels: a systematic review and metaanalysis. Diabetes Care 2010;33:1859-64.

10. American Diabetes Association. 8. Pharmacologic Approaches to Glycemic Treatment. Diabetes Care 2017;40(Suppl 1):S64-S74. 